\title{
PROCESS VALIDATION AND REGULATORY REQUIREMENTS OF METERED-DOSE INHALERS: AN OVERVIEW
}

\author{
ASHRANI SUNIL ${ }^{1 *}$, GOYAL ANJU², VAISHNAV RAJAT ${ }^{3}$ \\ ${ }^{1}$ Department of Pharmaceutical Quality Assurance, M. Pharma Research Scholar, B.N. Institute of Pharmaceutical Sciences, Udaipur, \\ Rajasthan, India. ${ }^{2}$ Department of Pharmaceutical Chemistry, B.N. Institute of Pharmaceutical Sciences, Udaipur, Rajasthan, India. \\ Email: sunilashrani93@gmail.com
}

Received: 05 September 2017, Revised and Accepted: 31 October 2017

ABSTRACT

The goal of the validation is to assure that quality is built into the system at every step, and not just tested for at the end. The validation activities will commonly include training on production material and operating procedures, training of people involved, and monitoring of the system while in production. Each and every doses form needs to be validated to reduce the chances of batch failures and market recalls. In case of metered-dose inhalers (MDIs) also it becomes mandatory. US Food and Drug Administration defines process validation as "establishing documented evidence which provides a high degree of assurance that a specific process will consistently produce a product meeting its predetermined specifications and quality characteristics." MDIs are known as device that is made to deliver a specific amount of aerosolized medication in the form of short burst directly to the lungs when inhaled by the patient. Furthermore, the inhalational drug delivery causes less pain and is convenient for administration. The patients of asthma, emphysema, and chronic obstructive pulmonary disease are prescribed for quicker relief. The present paper is a summary of process involved in the manufacturing of MDIs and focuses on the regulatory requirements along with their process validation.

Keywords: Metered-dose inhalers, Process validation, Chronic obstructive pulmonary disease, Bronchitis, etc.

(C) 2018 The Authors. Published by Innovare Academic Sciences Pvt Ltd. This is an open access article under the CC BY license (http://creativecommons. org/licenses/by/4. 0/) DOI: http://dx.doi.org/10.22159/ajpcr.2018.v11i2.22408

\section{INTRODUCTION}

During the past three to four decades, the pharmaceutical industry has gone leaps and bounds in context of providing better and improved medicines to the society. In earlier seventies, the initial emphasis on concept of validation commenced across the industry globally, and the manufactured products were tested for their compliance with their preestablished specifications, but soon, it was realized that final checking was not the scientific approach, and thus, they started checking each and every step of manufacturing the product [1]. Ted Byers and Bud Loftus were the first Food and Drug Administration (FDA) officials, in the mid-1970 who had proposed the concept of validation to improve the quality of pharmaceuticals. The goal of the validation is to assure that quality is built into the system at every step, and not just tested for at the end. The validation activities will commonly include training on production material and operating procedures, training of people involved, and monitoring of the system while in production [2].

US-FDA defines process validation as "establishing documented evidence which provides a high degree of assurance that a specific process will consistently produce a product meeting its predetermined specifications and quality characteristics." According to EMEA, Process validation can be defined as documented evidence that the process, operated within established parameters, can perform effectively and reproducibly to produce a medical product meeting its predetermined specifications and quality attributes [3]. Process validation has become a legal requirement by the cGMP and other regulatory bodies.

\section{IMPORTANCE OF PROCESS VALIDATION [4]}

- It provides assurance of quality of the manufactured products.

- Saves the time lapsed in remaking of the products.

- The process is optimized.

- Reduction in cost of quality.

- Nominal mix-ups and bottlenecks

- Reduces batch failures of the products and improves the productivity.

- Reduces market recall of products due to complaints of inferiority.

- Avoidance of capital expenditures.

\section{Validation team}

A multidisciplinary team is of prime requirement for conducting and supervising validation studies. Personnel who have training and experience in appropriate discipline may conduct and supervise such studies. The validation team comprises following members:

1. Head of quality assurance.

2. Head of engineering.

3. Validation manager.

4. Production manager.

5. Specialist validation discipline: All areas.

\section{Types of process validation [5]}

Prospective process validation

It is the process validation where an experimental plan known as validation protocol is implemented before the process is put to commercial use. Most of such validation efforts require some degree of prospective testing to generate validation support data.

\section{Concurrent process validation}

It is the process validation which provides documented evidence that the process is in a state of control when the process is actually implemented. This is normally performed by conducting in-process quality control tests during the manufacture of each production batch.

\section{Retrospective process validation}

It is the process validation where data's from previous experimentation taken from the records of the completed production batches are used to provide documented evidence that the process has been in a state of control before the request for such evidence.

\section{Revalidation}

Revalidation helps in providing the evidence that changes in a process and/or the process environment do not adversely affect process characteristics and product quality. 
Documentation [6,7]

Maintaining written record of each and everything going on in the company is very important activity as per the regulatory requirement by the authorities so cGMP, US-FDA, etc., all focus on good documentation practice. It is thus important for maintaining a record in process validation. The main documents required to be maintained are enlisted below:

I. Standard operating procedure (SOP)

II. Validation master plan

III. Validation protocol

IV. Validation report.

SOP

The SOP is a written document which clearly includes all the processing steps of the particular tasks and acts as evidence in case of conflicts of batch failures or market complains regarding any product.

The general format of the SOPs involves:

1. Title

2. Code

3. Objective

4. Scope

5. Definitions

6. Description

7. Safety

8. Documentation

9. Effective date, review date, and version number

10. Footer: Prepared by, reviewed by, approved by, and authorized by

11. References.

\section{Validation master plan}

The format and content should include:

- Introduction: Validation policy, scope, location, and schedule.

- Organizational structure: Personnel responsibilities. Plant/ process/product description: Rational for inclusions or exclusions and extent of validation.

- Specific process considerations that are critical and those requiring extra attention.

- Key acceptance criteria.

- Documentation format.

Table 1: MDI active ingredients and their quantities [14]

\begin{tabular}{lll}
\hline Type & Ingredient & Amount \\
\hline Propellants & CFCs 11, 12,114 & $60-90$ \\
Dispersing agents & Sorbitan trioleate & $0.01-0.8$ \\
& Sorbitan sesquioleate & $0.01-0.8$ \\
& Oleic acid & $0.01-0.8$ \\
& Soya lecithin & $0.01-0.1$ \\
Cosolvents & Ethyl alcohol & $2-38$ \\
Antioxidants and flavors & Water & $5-10$ \\
& Ascorbic acid & 0.1 \\
Antimicrobials & Saccharin & - \\
\hline
\end{tabular}

MDI: Metered-dose inhalers
- $\quad$ Reference to the required SOPs.

- Time plans of each validation project and subproject.

- List of products/processes/systems to be validated, summarized in a matrix format, and validation approach.

- $\quad$ Revalidation activities, actual status, and future planning [8].

\section{Validation protocol}

The validation protocol should be numbered, signed, and dated and should contain as a minimum the following information:

1. Title

2. Objective and scope

3. Responsibility

4. Protocol approval

5. Validation team

6. Product composition

7. Process flowchart

8. Manufacturing process

9. Review of equipments/utilities

10. Review of raw materials and packing materials review of analytical and batch manufacturing records

11. Review of batch quantities for validation (raw materials)

12. Review of batch quantities for validation (packing materials)

13. HSE requirements

14. Review of process parameters validation procedure

15. Sampling location

16. Documentation

17. Acceptance criteria

18. Summary

19. Conclusion

\section{Validation report}

The validation report should contain the approved validation protocol, tabulated or graphical results, process monitoring (forms), and all analytical results of the validation batches. The validation report should have a conclusion that explains the manufacturing specialist's statement and opinion stability testing on all validation batches must be performed according to the protocol, according to NDA/ANDA stability plan [9].

\section{METERED-DOSE INHALERS (MDIS)}

The origin of inhalation therapies dates back to $40^{\text {th }}$ century when in India people used to inhale the smokes of the Atropa belladonna plant for the prevention from cough. The evolution of this therapy and its effectiveness depends not only on a pharmacologically active molecule but also on the route of administration and its bioavailability [10].

MDIs therapy has become a prime therapy from the $20^{\text {th }}$ century with an advantage of small pocket-sized inhaler which is easy to carry for the patients. The aerosol delivery system depends on individual patient characterization and aerosol properties, i.e. distribution of particle, particle size, airway layout nature, etc. [11]

MDI products contain therapeutically active ingredients dissolved in a propellant, a mixture of propellants, or a mixture of solvents, propellants, and/or other excipients in compact pressurized aerosol dispensers. MDIs are known as a device that is made to deliver a

Table 2: Important valve tests per lot [14]

\begin{tabular}{lll}
\hline Test & Number of values & Characteristics \\
\hline Inspection of attributes & MIL-105E & Appearance, identity, proper assembly \\
Dimensional check & 40 & Proper components, identity \\
Valve delivery (spray weights) mean, RSD & 40 & Meter chamber size, in use test, meter chamber variability \\
Valve delivery at labeled number of actuations & 6 & Ruggedness of multiple sprays \\
Weight loss (leakage) & 12 & Sealing capability, proper rubber sealability \\
Loss of prime & 12 & Meter chamber sealability \\
Particulates & 12 & Valve cleanliness \\
Extractables & 12 & Rubber contaminants \\
\hline
\end{tabular}


specific amount of aerosolized medication in the form of short burst directly to the lungs when inhaled by the patient [12]. They are considered superior over oral drug delivery system because inhaled

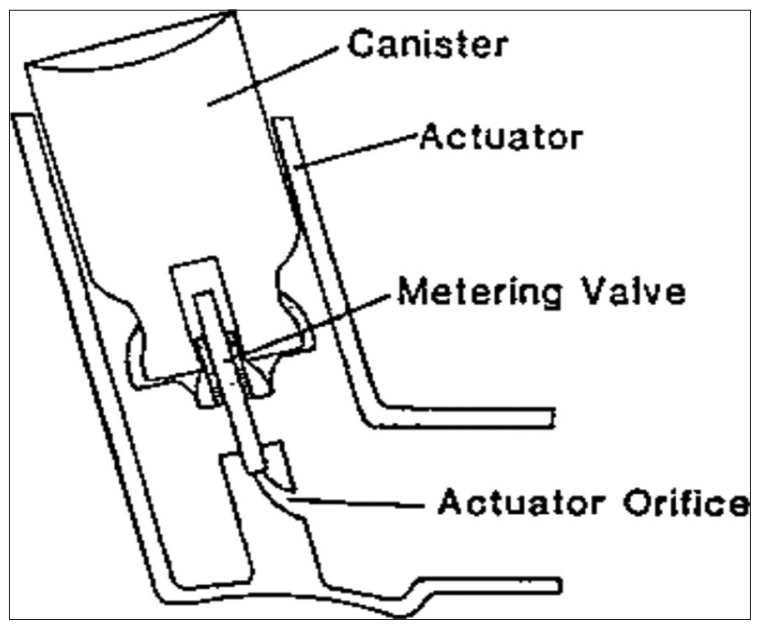

Fig 1: Schematic diagram of a metered-dose inhaler [14] beta-2 agonist bronchodilators produce a more rapid onset of action. Furthermore, the inhalational drug delivery causes less pain and is convenient for administration. The patients of asthma, emphysema, and chronic obstructive pulmonary disease are prescribed for quicker relief (Fig. 1) [13].

\section{REGULATORY REQUIREMENTS OF QUALITY SECTION OF MDIS AS PER EUROPEAN GUIDELINES [16]}

Pharmaceutical developments

- Moisture content: The test for moisture content is done and amount of moisture present is recorded to ensure stability of the product.

- Delivered dose: The test for delivered dose ensures the uniformity of dose delivered each time from the inhaler.

- Fine particle dose: The particle size distribution of the active substance is determined by impinge for fine particle dose.

- Use of spacer: When the spacer is used in some products its use should be validated and relevant information given in the summary product characters.

- Breath-actuated device: The actuated device is capable of triggering all target patient group must be demonstrated by proper data collection

- In use performance: The product must be easy to use by normal patients without sophistication.

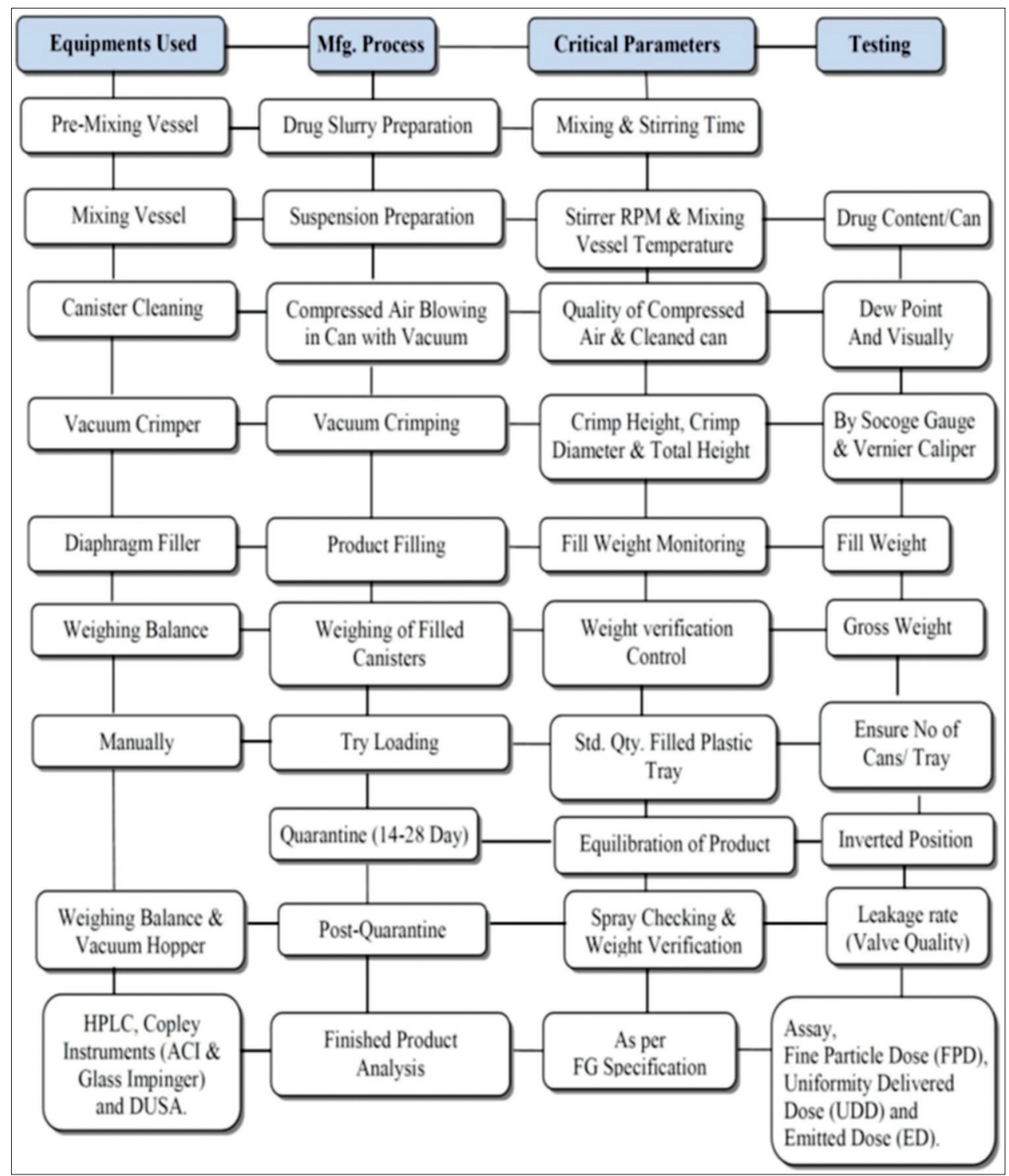

Fig 2: Schematic representation of manufacturing process of pressurized metered-dose inhalers [15] 
- Cleaning procedure: The cleaning procedure of the device should be clearly written described.

- Description of manufacturing process: The overall data of process validation of the process that demonstrates the validity of the process should be submitted.

- Control of excipients: The toxicity and purity data of excipient must be properly described.

\section{Control of drug product}

Moisture content: The test is performed if needed.

- Delivered dose uniformity: It is done to evaluate the uniformity of delivered dose.

- Leak rate: The leak rate test is done to optimize the leak within the acceptance criteria.

- Number of deliveries per inhaler: The number of deliveries per inhaler must be appropriate to meet within the labeled amount.

- Particulate matter: The absence of any particulate matter in the product must be ensured to increase the shelf life of the product.

- Container closure system: The specification for each component of the inhaler and its compliance with the specification for limits of leachable components and extraction studies should be given.

- Stability: The test for stability includes specification test, with the exception of the identity test and leachable moisture and microbial purity.

\section{Summary of product characteristics}

- Quantitative and qualitative composition: The qualitative and quantitative composition of the product should be properly stated.

- Posology and method of administration: The direction on how to use the inhaler and also the conditions in which one can opt for MDIs must be clarified.

- Special precaution for storage: The proper storage condition must be labeled on the product also if there is any special requirement should be also there.

\section{CONCLUSION}

Validation has been proven assurance for the process efficiency and sturdiness and it is the full-fledged quality attributing tool for the pharmaceutical industries. Validation eliminates the chances of batch failures as the products are manufactured as per preoptimization of each manufacturing steps. The conventional process of testing at last stage created much problems in maintain uniformity of each batch but with the introduction of the concept of process validation, it has been easy to maintain the batch uniformity of the product along with imparting quality in them. This paper has tried to summarize the process validation and regulatory requirements of MDIs which have emerged as a newer tool for drug delivery system with easy and quick response in providing relief to the asthmatic patients.

\section{AUTHORS CONTRIBUTION}

Sunil Ashrani: Compilation of data. Dr. Anju Goyal: Reviewing and checking of the manuscript.

\section{CONFLICTS OF INTEREST}

Nil.

\section{REFERENCES}

1. Nag S, Gouthami B, Madhuri L, Reddy LV, Krishnaveni N, Meyyanathan SN, et al. The concept of process validation in tablet manufacturing: Review. J Pharm Res 2012;5:1264-7.

2. Sandhya C, Bonthagarala B, Sai PD, Sivaiah KV. Process validation: An essential process in pharmaceutical industry. Int J Agric Sci Res 2015;1:179-82

3. Sharma M, Agarwal S, Agarwal S, Sharma M. Prospective validation: A review. Pharm Innov J 2015;4:1-7.

4. Nandhakumar L, Dharmamoorthy G, Rameshkumar S, Chandrasekaran S. An overview of pharmaceutical validation: Quality assurance view point. Int J Res Phys Chem 2011;1:1004-14.

5. Chaitanyakumar G, Rout RP, Ramtake S, Bhattacharya S. Process validation. Indian Pharm 2005;14-9.

6. U.S. Food and Drug Administration. Guideline on General Principles of Process Validation. Rockville, MD: U.S. FDA; 1987.

7. Akers J. Simplifying and improving process validation. J Parenter Sci Technol 2009;47:281-4.

8. Sharma S, Singh G. Process validation in pharmaceutical industry: An overview. J Drug Deliv Ther 2013;3:184-8.

9. Bai RR. Pharmaceutical validations. JPQA 2016;2:39-56.

10. Pattnaik SA, Niyogi P, Maharana L, Panigrahi L. Formulation, evaluation and solid-state thermographic characterization of CFC free beclometasone dipropionate pressurized metered dose inhalation. Int J Pharm Pharm Sci 2014;6:398-403

11. Rajendran R, Balan R, Ganesan N, Thiruvengadam D. Recent modalities in drug delivery via inhalation therapy-an advanced treatment strategy for pulmonary carcinoma. Int J Pharm Pharm Sci 2015;7:8-21

12. Kaur G, Rana AC, Seth N, Bala R. Process validation of metered dose inhaler: A review. Int Res J Pharm 2012;3:55-9.

13. Kumar GS, Gowrav MP. The newer dimension of inhalation aerosols in the prospective of validation. Int J Pharm Sci Res 2017;8:401-10.

14. Nash RA, Wachter AH. Pharmaceutical Process Validation. $3^{\text {rd }}$ ed. New York: Marcel Dekker, Inc.; 2003.

15. Musale VP, Jagdale SC. A review on validation of pharmaceutical inhalation aerosols-pressurized metered dose inhaler (pMDI). Int $\mathrm{J}$ Pharm Sci Res 2016;6:336-44

16. Shah D, Maheshwari D. Study of quality requirements of meter dose inhaler regulation in us and Europe. World J Pharm Pharm Sci 2015;4:623-34. 